\title{
Versorgungsstrukturgesetz 2012 - Was ändert sich tatsächlich?
}

\section{Zusammenfassung}

Das vieldiskutierte Versorgungsstrukturgesetz ist Ende des vergangenen Jahres vom Bun-destag beschlossen und vom Bundesrat gebilligt worden. Seit Jahresbeginn 2012 sind we-sentliche Teile des Gesetzes in Kraft. Im Großen und Ganzen entspricht das Gesetz dem bereits im Juni des vergangenen Jahres vorgelegten Referentenentwurf. Dennoch sind kurz vor der Beschlussfassung des Bundestages noch einige geplante Änderungen weggefallen und dafür andere hinzugetreten. Nachfolgend stellen wir die wesentlichen Änderungen im Krankenversicherungsrecht (SGB V) sowie im Zulassungswesen der Ärzte (ÄrzteZV) vor:

\section{Text}

\section{Delegation ärztlicher Leistungen ( $§ 28$ Abs. 1} Satz 2 AGB V)

Der Gemeinsame Bundesausschuss (G-BA) hat am 20.10.2011 die Richtlinie zur Heilkundeübertragung im Rahmen von Modellvorhaben und damit die Substitution ärztlicher Leistungen auf Angehörige der Kranken- und Altenpflegeberufe beschlossen. Die Neufassung des $\S$ 28 Abs. 1 Satz 2 SGB V sieht demgegenüber vor, dass die Partner der Bundesmantelverträge eine Liste von delegationsfähigen Leistungen erstellen sollen. Nach Auffassung des Gesetzgebers würden die bestehenden Möglichkeiten zur Delegation noch nicht in ausreichendem Umfang genutzt. Daher soll für die ambulante Versorgung beispielhaft festgelegt werden, bei welchen Tätigkeiten nichtärztliches Kranken- und Pflegepersonal ärztliche Leistungen erbringen kann, und welche Anforderungen an die Erbringung zu stellen sind. Grundsätzlich gilt zwar bei der ärztlichen Behandlung in der Gesetzlichen Krankenversicherung die Pflicht zur persönlichen Leistungserbringung. Das bedeutet allerdings nicht, dass der Arzt alle Leistungen in vollem Umfang eigenhändig erbringen muss. Vielmehr kann er ärztliche Leistungen an nichtärztliches Personal delegieren. Die Letztverantwortung des Arztes bleibt dabei - im Gegensatz zur Substitution - jedoch erhalten, was bedeutet, dass er die Hilfsperson je nach den Erfordernissen überwachen und anleiten muss.

\section{Entlassmanagement ( $\$ 39$ SGB V)}

Mit einer Ergänzung in § 39 SGB V wurde ein verbindlicher Anspruch der Versicherten auf das Entlassmanagement nach einem Krankenhausaufenthalt eingeführt. Der Gesetzgeber sah die bisherige Vorschrift als nicht ausreichend an, da es Probleme beim Übergang in die verschiedenen Versorgungsbereiche gegeben habe. Ziel des Entlassmanagements sei es, die Kontinuität der Versor- gung zu gewährleisten, die Kommunikation zwischen den beteiligten ambulanten und stationären Versorgungsbereichen zu verbessern sowie einen möglichen „Drehtüreffekt“ zu vermeiden. Daher sollen in die Verträge der Landesverbände der Krankenkassen und der Ersatzkassen mit der Landeskrankenhausgesellschaft oder mit den Vereinigungen der Krankenhausträger im Land zukünftig Einzelheiten zum Entlassmanage-ment festgelegt werden.

\section{Zuweisungsverbot ( $\$ 73$ Abs. 7 SGB V)}

Das bereits berufsrechtlich bestehende Verbot der Zuweisung gegen Entgelt ist jetzt auch in das SGB V aufgenommen worden. § 73 Abs. 7 SGB $\vee$ sieht eine $\S 31$ der ärztlichen Berufsordnung vergleichbare Regelung vor, wonach es Vertragsärzten nicht gestattet ist, für die Zuweisung von Versicherten ein Entgelt oder sonstige wirtschaftliche Vorteile sich versprechen oder sich gewähren zu lassen oder selbst zu versprechen oder zu gewähren.

Damit hat der Gesetzgeber klargestellt, dass ein Verstoß gegen das Zuweisungsverbot gleichzeitig immer auch einen Verstoß gegen vertragsärztliche Pflichten darstellt. Durch die Regelung soll insbesondere ermöglicht werden, im Rahmen der Zulassung von (Teil-) Berufsausübungsgemeinschaften zu prüfen, ob diese nicht allein zu dem Zweck gegründet wurden, unzulässige Zuweisungen gegen Entgelt oder sonstige wirtschaftliche Vorteile zu verschleiern.

\section{Honorarverteilung ( $\$ 87$ b SGB V)}

Das vertragsärztliche Vergütungssystem wird flexibilisiert und regionalisiert. Mit dem Versorgungsstrukturgesetz erhalten die Kassenärztlichen Vereinigungen wieder die Kompetenz, die Honorarverteilung eigenständig und nur noch im Benehmen mit den Krankenkassen festzulegen. Zugleich soll der Einheitliche Bewertungsmaßstab (EBM) durch eine bedarfsgerechtere Verteilung der Vergütungen 
schrittweise weiterentwickelt werden, um dem speziellen Versorgungsbedarf der Patienten und dem Leistungsspektrum der niedergelassenen Ärzte besser gerecht zu werden. Damit geht die Kompetenz für die Honorarverteilung von der Bundesebene auf die Landesebene über. Der Bewertungsausschuss wird zukünftig nur noch unverbindliche Empfehlungen abgeben. Die vertragsärztliche Vergütung erfolgt wieder im Rahmen eines regionalen Honorarverteilungsmaßstabs durch die Kassenärztlichen Vereinigungen, wobei die Trennung nach haus- und fachärztlichen Versorgungsbereichen beibehalten wird. Ab dem Jahr 2013 erhalten die Kassenärztlichen Vereinigungen auch bei der regionalen Verhandlung über die Höhe der Gesamtvergütung mit den Krankenkassen mehr Gestaltungsspielräume. Bis die Vergütungssystematik durch die Kassenärztlichen Vereinigungen umgesetzt worden ist, gelten die alten Regelleistungsvolumina allerdings noch fort.

Um weitere Anreize für eine vertragsärztliche Tätigkeit in unterversorgten Gebieten zu fördern, hat der Gesetzgeber in diesen Gebieten auf eine Mengenbegrenzung verzichtet und Leistungen von Maßnahmen zur Fallzahlbegrenzung und Fallzahlminderung ausgenommen. Zudem werden die Kassenärztlichen Vereinigungen verpflichtet, auf der Basis von Einzelfallprüfungen bei Bedarf weitergehende Ausnahmen vorzusehen (z. B. Ausnahme von der Abstaffelung des Punktwertes).

Darüber hinaus wird die Deckelung für extrabudgetäre Leistungen wieder aufgehoben, wodurch insbesondere das ambulante Operieren als ambulante Leistung gefördert werden soll.

\section{Beteiligung der medizinischen Fachgesellschaften vor der Neubewertung von Behandlungsmethoden durch den G-BA ( $\$ 92$ Abs. 7 d SGB V)}

Vor der Beschlussfassung zu einer nichtmedikamentösen Untersuchungs- oder Behandlungsmethode erhalten zukünftig neben den Ärztekammern auch die jeweils einschlägigen medizinischen Fachgesellschaften ein Recht zur Stellungnahme. Neben der bereits bisher auf der Grundlage der Verfahrensordnung erfolgenden Beteiligung der Fachkreise am Anfang eines neuen Beratungsverfahrens bedarf es auch vor der abschließenden Beschlussfassung im G-BA der Einbeziehung der einschlägigen Fachgesellschaften. Das Recht zur Stellungnahme und Anhörung soll nach dem Willen des Gesetzgebers die Gewähr dafür erhöhen, dass die Entscheidungen des GBA über neue Untersuchungs- und Behandlungsmethoden auf einer fachlich fundierten und die realen Versorgungsaspekte berücksichtigenden Grundlage getroffen werden. Maßgeblicher Ansprechpartner für den G-BA wird nach der zwischenzeitig bereits überarbeiteten Verfahrensordnung die Arbeitsgemeinschaft wissenschaftlicher medizinischer Fachgesellschaften (AWMF) sein.

\section{Medizinische Versorgungszentren - MVZ (§ 95 Abs. 1, 1 a, 6 SGB V)}

Bereits nach geltendem Recht handelt es sich bei Medizinischen Versorgungszentren um ärztlich geleitete Einrichtungen. Durch die Änderungen des Versorgungsstrukturgesetzes wird nunmehr vorgegeben, dass der ärztliche Leiter in dem Medizinischen Versorgungszentrum selbst als angestellter Arzt oder als Vertragsarzt tätig sein muss und in medizinischen Fragen keinen Weisungen unterliegen darf. Hierdurch soll die ärztliche Therapie- und Weisungsfreiheit gewährleistet werden.

Darüber hinaus werden die Gründungsvoraussetzungen für Medizinische Versorgungszentren neu gefasst. Künftig können Medizinische Versorgungszentren nur noch von zugelassenen Vertragsärzten und von zugelassenen Krankenhäusern gegründet werden. Die Gründungsberechtigung wird dadurch nach dem Willen des Gesetzgebers auf Leistungserbringer konzentriert, die den Großteil der ambulanten und stationären ärztlichen Versorgung der Versicherten leisten. Sonstige Leistungserbringer nach dem SGB V, die nicht an der vertragsärztlichen Versorgung teilnehmen, sind künftig nicht mehr berechtigt, Medizinische Versorgungszentren zu gründen. Eine Ausnahme gilt nur für gemeinnützige Trägerorganisationen. Zudem sind künftig als zulässige Rechtsformen für Medizinische Versorgungszentren nur noch Personengesellschaften, d.h. Gesellschaften bürgerlichen Rechts, Partnerschaftsgesellschaften oder Ärztegesellschaften nach der Musterberufsordnung der Ärzte sowie Gesellschaften mit beschränkter Haftung $(\mathrm{GmbH})$ zugelassen. Ziel des Gesetzgebers ist es, durch den Ausschluss von Aktiengesellschaften als zulässiger Rechtsform für Medizinische Versorgungszentren die Unabhängigkeit ärztlicher Entscheidungen von reinen Kapitalinteressen zu gewährleisten.

Allerdings gibt es einen umfassenden Bestandsschutz für Medizinische Versorgungszentren, die zum Zeitpunkt des Inkrafttretens der Neuregelung bereits zugelassen waren. Diese Zulassungen gelten unabhängig von der Rechtsform und der Trägerstruktur des bestehenden Medizinischen Versorgungszentrums unverändert fort. Eine Ausnahme von diesem umfassenden Bestandsschutz bildet lediglich die neue Voraussetzung, dass der ärztliche Leiter eines Medizinischen Versorgungszentrums auch in diesem tätig sein muss. Kommen bestehende Medizinische Versorgungszentren dieser Voraussetzung nicht bis zum 30.06.2012 nach, droht der Entzug der Zulassung.

\section{Rückumwandlung von Arztstellen (§ 95 Abs. 9 b SGB V i.V.m. § 32b Ärzte-ZV)}

Nach bisher geltendem Recht war die (Rück-)Umwandlung einer genehmigten Angestelltenstelle in einen eigenständigen Vertragsarztsitz nicht möglich. Zukünftig soll es 
Vertragsärzten ermöglicht werden, eine genehmigte Anstellung zu einem späteren Zeitpunkt in eine Zulassung umwandeln zu lassen. Hierzu sieht die Vorschrift vor, dass die genehmigte Anstellung vom Zulassungsausschuss auf Antrag des anstellenden Vertragsarztes in eine Zulassung umzuwandeln ist. Als Inhaber der bisherigen Arztstelle für einen angestellten Arzt kann der anstellende Vertragsarzt entscheiden, ob der bisher angestellte Arzt Inhaber der neuen Zulassung werden soll, oder ob er die Durchführung eines Nachbesetzungsverfahrens möchte, so dass er eine nicht mehr benötigte Arztstelle im Zuge des Nachbesetzungsverfahrens wirtschaftlich verwerten kann. Beantragt der anstellende Vertragsarzt keine Nachbesetzung hat der Zulassungsausschuss die Zulassung dem bisher angestellten Arzt zu erteilen. Hierdurch erhalten Vertragsärzte die flexible Möglichkeit, nach einer Bewährungsphase und bei entsprechendem Interesse zunächst angestellte Ärzte als Vertragsärzte gleichberechtigt in die Praxis zu integrieren. Diese Regelung gilt entsprechend für Medizinische Versorgungszentren, so dass auch ein Vertragsarzt, der in einem gesperrten Planungsbereich auf seine Zulassung verzichtet hat, um in einem Medizinischen Versorgungszentrum als angestellter Arzt zu arbeiten, die Angestelltenstelle zu einem späteren Zeitpunkt in eine Zulassung zurück umwandeln kann.

\section{Befristung von Zulassungen ( $\$ 98$ SGB V i.V.m. § 19 Ärzte-ZV)}

Durch die Änderungen des Versorgungsstrukturgesetzes wird erstmals die Möglichkeit geschaffen, Zulassungen zur vertragsärztlichen Versorgung zu befristen. Die Voraussetzungen für eine Befristung von Zulassungen werden in § 19 Abs. 4 der Zulassungsverordnung für Ärzte (Ärzte-ZV) geregelt. Diese Regelung soll nach der Auffassung des Gesetzgebers dem Ziel dienen, perspektivisch die Entstehung und Festschreibung von Überversorgung zu reduzieren. Daher ist eine Befristung von Zulassungen nur in offenen Planungsbereichen möglich, in denen der allgemeine bedarfsgerechte Versorgungsgrad mindestens 100 Prozent erreicht.

Über die Befristung einer Zulassung soll der Zulassungsausschuss nach pflichtgemäßem Ermessen entscheiden. Insoweit macht das Gesetz keine Vorgaben über den Befristungszeitraum; daher ist auch dieser nach pflichtgemäßem Ermessen des Zulassungsausschusses festzulegen. Dabei sollen neben den Versorgungsbedürfnissen die Interessen des zugelassenen Vertragsarztes berücksichtigt werden, da es inm möglich sein soll, die mit der Praxisgründung typischerweise verbundenen Investitionskosten während der Dauer der Zulassung zu refinanzieren. Daher hat der Zulassungsausschuss die Möglichkeit, die Befristung an das Erreichen eines bestimmten Lebensalters des Vertragsarztes oder das Ende der beruflichen Tätigkeit zu knüpfen.

\section{Bedarfsplanung ( $§ 99$ ff. SGB V)}

Den Beteiligten wird ein größerer gesetzlicher Gestaltungsspielraum für die Bedarfsplanung eingeräumt, um regionale Besonderheiten (z.B. regionale demografische Entwicklung oder Morbiditätsstrukturen) für eine bedarfsgerechte Versorgung berücksichtigen zu können. Zu diesem Zweck kann von der Bedarfsplanungsrichtlinie des G-BA abgewichen werden (z.B. abweichende Abgrenzungen der Planungsbereiche oder abweichende Verhältniszahlen für den bedarfsgerechten Versorgungsgrad).

Daneben wird in der Bedarfsplanung auch der mit dem Versorgungsstrukturgesetz eingeführten ambulanten spezialärztlichen Versorgung (vgl. § 116 b SGB V) Rechnung getragen. An der vertragsärztlichen Versorgung teilnehmende Ärztinnen und Ärzte, die spezialärztliche Leistungen erbringen, stehen eingedenk diese Leistungen nicht mehr im vollen Umfang zur Versorgung der Versicherten mit vertragsärztlichen Leistungen zur Verfügung, so dass diese Tätigkeit bei der Berechnung des Versorgungsgrades in Abzug zu bringen ist. Der G-BA erhält deshalb den Auftrag, in der Bedarfsplanungsrichtlinie Bestimmungen über den Umfang der Berücksichtigung dieser Tätigkeit zu treffen.

Umgekehrt sollen zukünftig neben zugelassenen und bei einem Vertragsarzt oder in einem Medizinischen Versorgungszentrum angestellte Ärzte alle an der vertragsärztlichen Versorgung teilnehmenden Ärzte in der Bedarfsplanung erfasst werden. Daher hat der G-BA den Auftrag, in der Bedarfsplanungsrichtlinie Bestimmungen zur Berücksichtigung auch der durch Ermächtigung an der vertragsärztlichen Versorgung teilnehmenden Ärzte bei der Berechnung des Versorgungsgrades zu treffen. Dies betrifft sowohl Ärztinnen und Ärzte, die über eine individuelle Ermächtigung verfügen (z.B. nach $\S 116$ ermächtigte Krankenhausärzte) als auch Ärztinnen und Ärzte, die in einer ermächtigten Einrichtung (z.B. in einem Krankenhaus, einer Psychiatrischen Institutsambulanz oder einem sozialpädiatrischen Zentrum) tätig sind.

Letztlich wird die bisherige Regelung, die vorschreibt, dass die regionalen Planungsbereiche den Stadt- und Landkreisen entsprechen sollen, durch eine flexiblere Regelung ersetzt. Daher ist es künftig auch möglich, bei der Größe der Planungsbereiche nach Arztgruppen oder auch nach hausärztlicher, allgemeiner fachärztlicher und spezialisierter fachärztlicher Versorgung zu differenzieren. Diese Neuregelung soll zum 1. Januar 2013 wirksam werden.

\section{Vorkaufsrecht von Zulassungen durch die $K^{`}$ 'en (§ 103 SGB V)}

Für viele Diskussionen hat bereits die Ankündigung gesorgt, den Kassenärztlichen Vereinigungen die Möglichkeit einzuräumen, nach Beendigung eines Ausschreibungs- 
verfahrens zur Nachbesetzung eines Vertragsarztsitzes ein Vorkaufsrecht auszuüben. Zwar sieht auch die endgültige Fassung des Versorgungsstrukturgesetzes ein solches Vorkaufsrecht der Kassenärztlichen Vereinigungen vor, allerdings erhält der Zulassungsausschuss die Aufgabe, im Vorfeld über die Nachbesetzung eines Vertragsarztsitzes in einem Planungsbereich, in dem wegen Überversorgung Zulassungsbeschränkungen angeordnet sind, zu entscheiden.

Endet künftig die Zulassung eines Vertragsarztes in einem Planungsbereich, für den Zulassungsbeschränkungen angeordnet sind, durch Tod, Verzicht oder Entziehung und soll die Praxis von einem Nachfolger weitergeführt werden, haben der Vertragsarzt oder seine Erben einen Antrag beim Zulassungsausschuss auf Durchführung eines Nachbesetzungsverfahrens zu stellen. Liegt dem Zulassungsausschuss ein Antrag auf Durchführung eines Nachbesetzungsverfahrens vor, hat er zu prüfen, ob auf eine Nachbesetzung des Vertragsarztsitzes aus Versorgungsgründen verzichtet werden kann. Im Rahmen seiner Prüfung hat der Zulassungsausschuss jedoch auch wirtschaftliche Gesichtspunkte zu berücksichtigen. Hat er z.B. darüber zu entscheiden, ob ein Vertragsarztsitz innerhalb einer Berufsausübungsgemeinschaft nachbesetzt werden soll, sind auch die Auswirkungen seiner Entscheidung auf die Berufsausübungsgemeinschaft zu berücksichtigen. Haben der Vertragsarzt oder seine Erben dargelegt, dass sich als Praxisnachfolger eine oder mehrere Personen bewerben werden, bei denen es sich um den Ehegatten, Lebenspartner bzw. ein Kind oder um einen angestellten Arzt des bisherigen Vertragsarztes handelt, muss ein Nachbesetzungsverfahren durchgeführt werden.

Im Übrigen kann der Zulassungsausschuss das Unterlassen eines Nachbesetzungsverfahrens nur mit der Mehrheit der Stimmen im Zulassungsausschuss beschließen. Hat der Zulassungsausschuss beschlossen, kein Nachbesetzungsverfahren durchzuführen, hat die Kassenärztliche Vereinigung dem Vertragsarzt oder den Erben eine Entschädigung zu zahlen. Allerdings wird aufgrund der paritätischen Besetzung des Zulassungsausschusses mit je drei Vertretern der Ärzte und der Kassen in der Regel nicht mit der Ablehnung der Durchführung eines Nachbesetzungsverfahrens zu rechnen sein.

\section{Beratung vor Regress ( $§ 106$ Abs. 5 e SGB V)}

Bei erstmaliger Überschreitung des Richtgrößenvolumens um mehr als 25 v. H. soll zukünftig zunächst kein Regress festgesetzt werden, bevor den betroffenen Vertragsärzten nicht zuvor zumindest eine einmalige Beratung angeboten wurde. Damit das wirtschaftliche Risiko infolge einer Überschreitung des Richtgrößenvolumens kalkulierbar bleibt und insoweit Rechtssicherheit besteht, wird Vertragsärzten dabei zudem die Möglichkeit eingeräumt, in begründeten Fällen bereits im Rahmen dieser Beratung eine Feststellung der Prüfungsstelle über die Anerkennung von Praxisbesonderheiten zu beantragen. Ein Feststellungsinteresse kann beispielsweise für geltend gemachte Praxisbesonderheiten bestehen, die nicht vorab anerkannt oder bereits Gegenstand von Prüfungsverfahren gewesen sind.

\section{Vor- und Nachstationäre Behandlung ( $\$ 115$ a SGB V)}

§ 115 a SGB V richtet sich an die Krankenhäuser und regelt die gesetzlichen Voraussetzungen der vor- und nachstationären Behandlung im Krankenhaus und deren Abgrenzung zur vertragsärztlichen Versorgung. Die Auslagerung von Leistungen auf niedergelassene Vertragsärzte durch Krankenhäuser im Rahmen der vor- und nachstationären Behandlung des Krankenhauses nach § 115 a war bereits bisher auf der Grundlage einer ausdrücklichen Beauftragung des niedergelassenen Vertragsarztes durch das behandelnde Krankenhaus möglich. Bei der Neuregelung handelt es sich daher um eine gesetzliche Klarstellung zur Flexibilisierung der Zusammenarbeit von Krankenhäusern und Vertragsärzten im Rahmen von Kooperationen bei der vor- und nachstationären Behandlung.

\section{Ambulante Operationen im Krankenhaus ( $\S$ 115 b SGB V)}

Nach der Rechtsprechung des Bundessozialgerichts (BSG, Urteil vom 24. März 2011 - B 6 KA 6/10 R -) ließ die bisherige Regelung zum ambulanten Operierens im Krankenhaus nach $\S 115 \mathrm{~b}$ in Verbindung mit dem dreiseitigen AOP-Vertrag nur zu, dass ambulante Operationen durch Operateure des Krankenhauses oder durch Belegärzte, jeweils in Verbindung mit einem Anästhesisten des Krankenhauses, erbracht werden. Danach durften weder niedergelassene Vertragsärzte ohne Belegarztstatus ihre Patienten ambulant im Krankenhaus operieren noch durften Krankenhäuser zur Durchführung ihrer ambulanten Operationen solche Ärzte einsetzen. Insbesondere waren Vereinbarungen zwischen Krankenhäusern und niedergelassenen Vertragsärzten mit dem Ziel, solche Kooperationen zu ermöglichen, nach der Rechtsprechung des Bundessozialgerichts mit der geltenden Rechtslage nicht vereinbar.

Da der Gesetzgeber auch in diesem Bereich eine Flexibilisierung der Zusammenarbeit von Krankenhäusern und Vertragsärzten herbeiführen möchte, hat er nun eine ausdrückliche gesetzliche Vorgabe an die Parteien des AOP-Vertrages aufgenommen, wonach ambulant durchführbare Operationen im Krankenhaus auch auf der Grundlage derartiger vertraglicher Kooperationen des Krankenhauses mit niedergelassenen Vertragsärzten erfolgen können. Vertragsarztrechtliche Vorschriften stehen einer solchen Regelung nun nicht mehr entgegen. Daher ist es zukünftig möglich, ambulante Operationen im Krankenhaus auch vor Vertragsärzten auf der Grundlage von Honorararztverträgen erbringen zu lassen. Diese 
Neuregelung lässt allerdings offen, ob auch stationäre Krankenhausleistungen durch Honorarärzte erbracht werden dürfen. Daher ist bei stationären Leistungen bis zu einer Entscheidung des BSG beim Einsatz von Honorarärzten Zurückhaltung geboten.

\section{Ambulante spezialfachärztliche Versorgung ( $\S$ 116 b SGB V)}

An die Stelle des bisherigen $\S 116$ b SGB V (Ambulante Behandlung im Krankenhaus) tritt eine völlig neu konzipierte ambulante spezialfachärztliche Versorgung. Damit soll stufenweise eine ambulante spezialfachärztliche Versorgung für Erkrankungen mit besonderen Krankheitsverläufen, seltenen Erkrankungen und hochspezialisierten Leistungen als eigenständiger Versorgungsbereich sowohl für niedergelassene Vertragsärzte als auch für Krankenhausärzte unter gleichen Qualitäts- und Vergütungsbedingungen eingeführt werden.

Nach heftiger Kritik an der im Referentenentwurf vorgesehenen Regelung hat der Gesetzgeber die Regelung noch einmal entsprechend überarbeitet. In dem Bereich der ambulanten spezialfachärztlichen Versorgung sollen seltene Erkrankungen und Erkrankungen mit schweren Verlaufsformen wie Krebs, HIV/AIDS, Mukoviszidose und Multiple Sklerose behandelt werden, wobei die Versorgung sowohl in der Praxis des Vertragsarztes als auch im Krankenhaus ambulant erfolgen kann. Nicht erfasst sind dagegen ambulante Operationen und stationsersetzende Eingriffe. Voraussetzung für die spezialfachärztliche Behandlung am Krankenhaus ist ein vertragsärztlicher Überweisungsvorbehalt, der jedoch noch der Ausgestaltung durch den Gemeinsamen Bundesausschuss bedarf.

Für die Vergütung der Leistungen soll eine Kalkulationssystematik mit diagnosebezogenen Gebührenpositionen in Euro vom Spitzenverband Bund der Krankenkassen, der Deutschen Krankenhausgesellschaft und der Kassenärztliche Bundesvereinigung gemeinsam eingeführt werden. Bis dahin erfolgt die Vergütung vorläufig nach dem Einheitlichen Bewertungsmaßstab (EBM). Die Abrechnung erfolgt direkt über die Krankenkassen. Vertragsärzte können stattdessen auch über die Kassenärztliche Vereinigung abrechnen.

Die Rahmenbedingungen für die spezialfachärztliche Versorgung soll der Gemeinsame Bundesausschuss bis zum 31.12.2012 festlegen. Darin sollen für alle Leistungserbringer einheitliche Anforderungen sowie besondere Maßnahmen zur Qualitätssicherung enthalten sein. Für die Entscheidung über die Teilnahme an der spezialfachärztlichen Versorgung ist zukünftig nicht mehr die Bezirksregierung, sondern ein um Vertreter der Landeskrankenhausgesellschaften erweiterter Landesausschuss zuständig. Für eine Übergangszeit von zwei Jahren gelten die den Krankenhäusern bereits erteilten Genehmigungen nach $\S 116$ b SGB V fort. Danach erfolgt die Teilnahme durch die entsprechende Anzeige bei dem zuständigen Landesausschuss, sofern die gesetzlichen Vorgaben erfüllt sind.

\section{Beteiligung an Unternehmen von Leistungserbringern (§ 128 SGB V)}

Die Regelungen zur Zusammenarbeit zwischen ärztlichen und nichtärztlichen Leistungserbringern haben eine weitere Verschärfung erfahren. Danach stellen nunmehr auch Einkünfte aus Beteiligungen an Unternehmen von Leistungserbringern, die Vertragsärzte durch ihr Verordnungs- oder Zuweisungsverhalten selbst maßgeblich beeinflussen, unerlaubte Zuwendungen dar. Nach Auffassung des Gesetzgebers sei diese Änderung notwendig gewesen, um zu verhindern, dass Vertragsärzte das Zuwendungsverbot durch Beteiligung an Unternehmen von Leistungserbringern im Hilfsmittelbereich umgehen. Darüber hinaus hat der Gesetzgeber nunmehr auch klargestellt, dass Zuwiderhandungen gegen die Verbote des $\S 128$ SGB V einen Verstoß gegen vertragsärztliche Pflichten darstellen, von dem die zuständige Kassenärztliche Vereinigung zu unterrichten ist.

\section{Bewertung von Behandlungsmethoden - Erprobungsphase ( $§ 137$ c SGB V)}

Der Ausschluss einer bestimmten Untersuchungs- oder Behandlungsmethode aus der stationären Krankenhausversorgung kann grundsätzlich nur dann erfolgen, wenn nach Feststellung des G-BA die überprüfte Methode kein Potenzial als erforderliche Behandlungsmethode in der stationären Versorgung bietet. Ein Potenzial für eine Erforderlichkeit kann sich etwa daraus ergeben, dass die Methode aufgrund ihres Wirkprinzips und der bisher vorliegenden Erkenntnisse mit der Erwartung verbunden ist, dass andere aufwändigere, für den Patienten invasivere oder bei bestimmten Patienten nicht erfolgreiche Methoden ersetzt werden können, die Methode weniger Nebenwirkungen hat, sie eine Optimierung der Behandlung bedeutet oder die Methode in sonstiger Weise eine effektivere Behandlung ermöglichen kann. Die Feststellung, dass der Nutzen der Methode für eine Anerkennung noch nicht hinreichend belegt ist, reicht dagegen nicht aus, um sie unmittelbar aus der Krankenhausversorgung auszuschließen.

Ist daher der Nutzen der überprüften Methode für eine positive Anerkennung nicht hinreichend belegt, weist sie aber das Potenzial als erforderliche Behandlungsalternative auf, so hat der G-BA zukünftig eine Richtlinie zur Erprobung zu beschließen.

\section{Wegfall der Residenzpflicht (§ 24 Ärzte-ZV)}

Bislang waren Vertragsärzte verpflichtet, ihren Wohnort so wählen, dass sie auch in sprechstundenfreien Zeiten ihren Vertragsarztsitz in angemessener Zeit erreichen konnten, wenn dies zur Versorgung der Versicherten er- 
forderlich war. Diese Verpflichtung wurde ersatzlos gestrichen, so dass der Wohnort nunmehr unabhängig von Praxissitz gewählt werden kann. Die Aufhebung dieser sogenannten Residenzpflicht lässt jedoch die Regelungen zur Teilnahme am organisierten Notdienst unberührt.

\section{Vereinfachung der Eröffnung von Zweigpraxen (§ 24 Ärzte-ZV)}

Künftig wird es leichter sein eine Zweigpraxis zu eröffnen. Der Gesetzgeber hat die Voraussetzungen für die Eröffnung von Zweigpraxen etwas weicher gestaltet, da nun auch eine geringfügige Beeinträchtigung der Versorgung am Ort des Vertragsarztsitzes unbeachtlich ist, wenn sie durch die Verbesserung der Versorgung an dem weiteren Ort aufgewogen werden kann. Durch diese Änderung sollen die Zulassungsausschüsse stärker als bisher dazu angehalten werden, bei der Genehmigungsentscheidung die Versorgungssituation an beiden Tätigkeitsorten zu berücksichtigen und den Versorgungsbedarf am Ort der Zweigpraxis ins Verhältnis zu einer eventuellen Beeinträchtigung der Versorgung am Vertragsarztsitz zu setzen. Je größer der Versorgungsbedarf am Ort der Zweigpraxis sei, desto eher seien Beeinträchtigungen der Versorgung am Vertragsarztsitz hinzunehmen, so die Gesetzesbegründung. Durch die Ergänzung erhielten die Zulassungsausschüsse die nötige Flexibilität, um die Eröffnung von Zweigpraxen vor allem dort zu genehmigen, wo dies zur Sicherstellung einer bedarfsgerechten Versorgung und zur Vermeidung von Unterversorgung besonders sinnvoll sei.

\section{Erschwerung der Sitzverlegung (§ 24 Ärzte-ZV)}

Umgekehrt wird es in Zukunft jedoch erschwert, den Praxissitz zu verlegen. Das bisherige Recht gewährte einem Vertragsarzt die Möglichkeit, seinen Vertragsarztsitz innerhalb eines Bedarfsplanungsbezirks zu verlegen, wenn Gründe der vertragsärztlichen Versorgung dem nicht entgegenstehen. Mit der Novellierung stellt der Gesetzgeber nun klar, dass die Zulassungsausschüsse bei der Prüfung eines Antrages auf Verlegung des Vertragsarztsitzes vorrangig darauf zu achten haben, dass Versorgungsgesichtspunkte einer Verlegung des Vertragsarztsitzes nicht entgegenstehen. Führt damit z.B. die Verlegung eines Vertragsarztsitzes in einen anderen Stadtteil zu Versorgungsproblemen in dem Stadtteil, in dem sich der Vertragsarztsitz bisher befand, hat der Zulassungsausschuss den Verlegungsantrag abzulehnen.

\section{Gewinnverteilung in Teil-Berufsausübungsgemeinschaften ( $\$ 33$ Ärzte-ZV)}

In § 33 Ärzte-ZV wurde das bereits in den Berufsordnungen enthaltene Verbot aufgenommen, dass Teil-Berufsausübungsgemeinschaften nicht mit dem Ziel einer Umgehung des Verbots der Zuweisung von Versicherten gegen Entgelt gegründet werden dürfen. Die gemeinsame Berufsausübung, bezogen auf einzelne Leistungen, ist daher nur dann zulässig, wenn sie nicht einer Umgehung des Verbots der Zuweisung von Versicherten gegen Entgelt oder sonstige wirtschaftliche Vorteile dient. Dies betrifft insbesondere Teil-Berufsausübungsgemeinschaften mit Ärzten, deren Beitrag sich auf das Erbringen medizinisch-technischer Leistungen auf Veranlassung der übrigen Mitglieder der Berufsausübungsgemeinschaft beschränkt. Ebenso unzulässig ist die Verteilung von Gewinnen aus der gemeinsamen Berufsausübung in Abhängigkeit von Zuweisungszahlen. Entscheidend ist bei solchen Berufsausübungsgemeinschaften daher, dass die Gewinnverteilung der tatsächlichen Leitungserbringung des jeweiligen Vertragsarztes entspricht. Durch die Aufnahme dieser Regelung in die Zulassungsverordnung soll es zukünftig dem Zulassungsausschuss möglich sein, Teil-Berufsausübungsgemeinschaften, die gegen dieses Verbot verstoßen, die Genehmigung zu versagen bzw. die Genehmigung mit Auflagen zu versehen.

Rechtsanwalt Dr. A. Wienke

Fachanwalt für Medizinrecht

Rechtsanwältin A. Stenger

LL.M. Medizinrecht

\section{Korrespondenzadresse:}

Dr. iur. Albrecht Wienke

Wienke \& Becker - Köln, Bonner Straße 32350968 Köln Awienke@Kanzlei-WBK.de

\section{Bitte zitieren als}

Wienke A, Stenger A. Versorgungsstrukturgesetz 2012 - Was ändert sich tatsächlich? GMS Mitt AWMF. 2012;9:Doc5.

DOI: 10.3205/awmf000253, URN: urn:nbn:de:0183-awmf0002538

Artikel online frei zugänglich unter

http://www.egms.de/en/journals/awmf/2012-9/awmf000253.shtml

Eingereicht: 31.01 .2012

Veröffentlicht: 01.02.2012

Copyright

(C2012 Wienke et al. Dieser Artikel ist ein Open Access-Artikel und steht unter den Creative Commons Lizenzbedingungen ( $h$ ttp://creativecommons.org/licenses/by-nc-nd/3.0/deed.de). Er darf vervielfältigt, verbreitet und öffentlich zugänglich gemacht werden, vorausgesetzt dass Autor und Quelle genannt werden. 